\title{
Colour specification at the design to production interface
}

\section{Document Version}

Accepted author manuscript

Link to publication record in Manchester Research Explorer

\section{Citation for published version (APA):}

Oulton, D., \& Young, T. (2004). Colour specification at the design to production interface. International journal of clothing science and technology, 16(1/2), 274-284.

\section{Published in:}

International journal of clothing science and technology

\section{Citing this paper}

Please note that where the full-text provided on Manchester Research Explorer is the Author Accepted Manuscript or Proof version this may differ from the final Published version. If citing, it is advised that you check and use the publisher's definitive version.

\section{General rights}

Copyright and moral rights for the publications made accessible in the Research Explorer are retained by the authors and/or other copyright owners and it is a condition of accessing publications that users recognise and abide by the legal requirements associated with these rights.

\section{Takedown policy}

If you believe that this document breaches copyright please refer to the University of Manchester's Takedown Procedures [http://man.ac.uk/04Y6Bo] or contact uml.scholarlycommunications@manchester.ac.uk providing relevant details, so we can investigate your claim.

\section{OPEN ACCESS}




\section{IJCST}

$16,1 / 2$

\section{4}

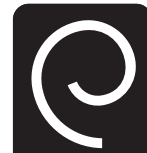

Emerald

International Journal of Clothing Science and Technology

Vol. 16 No. 1/2, 2004 pp. $274-284$

(c) Emerald Group Publishing Limited 0955-6222

DOI $10.1108 / 09556220410520540$

\section{Colour specification at the design to production interface}

David P. Oulton

Department of Textiles, UMIST, Manchester, UK

Tara Young

Worthington Manufacturing Ltd, Cheshire, UK

Keywords Colours technology, Textile manufacturing processes

Abstract This paper describes how the communication of colour specifications between designers and technical production personnel has been improved using calibrated colour and digital networking. The electronic colour communication system known as "Imagemaster" ${ }^{2 M}$ is described in which both colour and texture are quantified by calibrated variables. Colour is calibrated by reference to CIE colour co-ordinates. Imagemaster also uses and if necessary generates a reflectance curve for each object on the screen for use as a production colour specification. A novel colorimetric model of textile textures based on image content is described, which can be used to predict the independent effect of texture as a distinct component of overall colour appearance. Close electronic collaboration between all those contributing to design, product development and production is described. Savings in the complexity, cost and lead-time for achieving correct colour and technical specifications are reported.

\section{Introduction}

Colour plays a vital part in the development, marketing, and sales success of a wide range of products. In technical terms, it is an accepted practice to work to production colour tolerances of less than delta $E 2$ (CIE $L * a * b *)$ under three different illuminants, and colour management systems have been developed in textile manufacture and retailing, which enable production processes to be controlled to this level (Berns, 2000).

These and other technicalities of Industrial Colour Management are however not normally the concern of the designer.

Design inspiration, and design development are essentially visual and creative processes. The design process has its own distinct language and internal communication methods. Designers also have a different perspective from technologists on visual acceptability once the design has been reproduced, perhaps by industrial dyeing or printing.

There are thus readily apparent differences of approach to colour specification and colour-match acceptability between designers and production technologists. They often cause extra expense in sampling, time delays, and unnecessary compromises in the final product. In other words, we have an interface problem.

The Imagemaster system developed at UMIST (Oulton and Porat, 1997) seeks to bridge the "communication gap" between the two separate worlds of design and production. The designers and technologists must of course be 
given the tools to construct suitable messages to resolve the communication problem. On one side, there are tools concerned with manipulating and visualizing colour appearance and texture, and the message developed is primarily visual. On the other side, the tools are concerned with available dyes, dye recipes and production colour specifications. The specifications are then represented by measured and calibrated numbers (including the colour).

The first requirement is to make it easy to create dye recipes and also easy to create garment or fabric visualizations that look exactly like the ultimate product. The second is to make them readily inter-convertible. The designer can generate a technical message consisting of numeric specifications accompanied by a visual image of what is needed in terms of colour and texture. The production technologist can visualize and specify the colour and texture that might be produced by his chosen dye recipe (Oulton et al., 1996).

\section{Precise colour communication}

Two key technical developments enable the visualisation and communication of colour specifications. The first is the colour calibration of a computer monitor screen. A link must be established between visual colour appearance and its technical specification by CIE co-ordinates. The system developed at UMIST establishes on-screen colour reproduction of CIE specifications to better than delta $E 1$ (mean) CIE $L * a * b * \mathrm{D}_{65}$ colour difference for all reproducible on-screen colours. The calibration system requires at most 30 calibration points and can be run in under 5 min (Oulton and Porat, 1991).

The second major advance in colour communication is the use of both measured and synthetic reflectance curves to communicate full colour specifications digitally (Hawkyard, 1993). This method is now regularly used to transmit production colour specifications electronically to remote production units. At the receiving site, the reflectance curve is used as input to a local colourant recipe formulation computer system, which reproduces the desired colour to delta $E 1$ or better under multiple illuminants (i.e. a close reflectance curve match).

\subsection{The accuracy of on-screen colour reproduction}

Colour calibration by the UMIST “Adaptive Driver" system (Oulton and Porat, 1991) delivers local, device dependent RGB gun-drive values. These are generated by a dynamic non-linear three-dimensional transform, from CIE colour co-ordinate definitions, whose content is derived by establishing the direction, scaling, and non-linearity of the vectors of CRT colour reproduction space. Colour calibration is based on feed-back measurement of screen colour using a Minolta CA100 colour analyser under system control. The CRT analyser is used to feed the CIE co-ordinates of a screen colour back to the calibration software allowing an unique mapping to be built between independent $\mathrm{CIE}$ co-ordinates and device RGB co-ordinates. A typical 
IJCST

$16,1 / 2$

276

calibration process takes approximately $5 \mathrm{~min}$ and uses not more than 30 calibration points on the CIE co-ordinate grid of 16.77 million possible on-screen colour specifications.

Long term exhaustive trials have shown that across the 16 million plus colours, the system is capable of reproducing the desired CIE colour specifications to within an average of 0.5 delta $E$ (CMC 2:1). Table I shows the mean reproduction errors after sampling at $5^{\circ}$ hue intervals. For each hue interval, all possible combinations of Chroma and Lightness based on reproduction of CIE specified colours at five unit intervals of $L, C$ and $H^{\circ}$, have been measured and re-measured over varying time periods.

Coverage of CRT colour-space included all colour specifications where one or more gun drive values reach the range limits of 0-255.

$\mathrm{R}, \mathrm{G}$ and $\mathrm{B}$ gun-drive values are integers in the range $0-255$. This produces stepwise colour change on a grid of 16.77 million possible colours. A real number CIE specification may convert exactly into an integer gun-drive value, or it may be rounded to the nearest integer. This rounding process produces quantisation errors which can reach CMC 2:1 delta $E$ values of 0.6. The calibration routine thus appears to be generating gun-drive values which are accurate to the nearest integer value in most cases.

The Minolta CA 100 instrument used to measure screen patches in both calibration and testing is a Tristimulus instrument. It therefore gives a potentially inaccurate determination of screen chromaticity. The specific instrument used in the above-mentioned test, has been cross calibrated against a Bentham Tele-radio-spectrophotometer. Relatively minor smooth distortion of chromaticity was found across colour-space.

\section{Precision colour imaging}

Having put in place the essential elements of colour communication, the UMIST Colour Communication Research Group addressed the problem of modelling the complex surface texture of textile materials. This required the development of computer systems to capture, store, process, and analyse CIE specified colour images typically at 3,000 $\times 2,000$ pixel resolution, often derived from 24 bit RGB originals.

\begin{tabular}{lccccccc}
\hline & $\begin{array}{c}\text { Min. error } \\
\text { (DE }\end{array}$ & $\begin{array}{c}\text { Max. error } \\
\text { (DE CMC }\end{array}$ & \multicolumn{2}{c}{$\begin{array}{c}\text { Mean error } \\
\text { (DE CMC }\end{array}$} & \multicolumn{2}{c}{ Mean differences } & \multicolumn{2}{c}{$\begin{array}{c}\text { Number of } \\
\text { samples }\end{array}$} \\
Co-ordinate set & CMC2:1) & $2: 1)$ & $2: 1)$ & $h$ & $c$ & $I$ & measured \\
\hline $\begin{array}{l}\text { Example hue } \\
\text { page }\left(H=30^{\circ}\right)\end{array}$ & 0.025 & 1.348 & 0.487 & 0.658 & 0.794 & 0.097 & 1,908 \\
$\begin{array}{l}\text { Full set of } 72 \\
\text { hue pages }\end{array}$ & 0.01 & 1.969 & 0.484 & 0.650 & 0.792 & 0.148 & 124,968 \\
\hline
\end{tabular}


The objectives are to:

(1) enable early prototyping decisions to be based on colorimetrically accurate photographic images of potential products and product colours;

(2) enable "CAD conferencing" in close buyer-supplier partnerships, and across supplier production sites;

(3) add surface texture simulation to the colour communication process, without sacrificing any precision in the colour specification.

\section{Visualisation of colour in context}

Visual testing has revealed that a "colour in context" format is required, for a correct visualisation of $\mathrm{CIE}$ co-ordinate specified colour. The default display environment used is an image simulating $\mathrm{D}_{65}$ illumination in a standard matching booth. Alternative environments can be constructed on-screen, and the visual effect can be simulated of any alternative illuminant that has a known spectral power distribution (SPD).

An important principle in on-screen "virtual product" visualization is the elimination of electronic menus and windowing features from the field of view during colour judgement. Information screens and tools are therefore only displayed and accessed (from pop-up menus) as and when required.

The system has been tested both industrially and in the lab (Oulton and Porat, 1991; Oulton et al., 1996; Smith, 1999) for visual match quality between physical samples in the matching booth, and the on-screen simulation of the measured CIE co-ordinates. The quality of visual match is rated high enough to communicate an accurate visualisation of a measured CIE colour specification under a range of alternative illuminants.

\section{Design-led colour and appearance modification}

Single colours, colour ranges and colour libraries can be built up from measured instrumental input, numerical data input, or established visually on the computer screen. The visual colour manipulation tools pay particular attention to the properties of colour, and use intuitive techniques to locate colours in colour space. Internal functions convert 2D mouse input co-ordinates into colour visualizations and vice versa.

When new colours are generated on screen, both CIE co-ordinates and a synthetic spectral curve are generated for use as the master colour definitions. Alternatively, a spectrophotometer can be interfaced to the system, and used to generate the spectral data from a physical sample of the colour.

\subsection{User interaction with colour definitions}

An excess of 1 million colours are frequently present in an image. The software logically groups these into on-screen sets, each of which is a distinct object simulation. Each such object can then be manipulated independently, both in colour and position on the screen. The users can therefore build-up complex 
IJCST

$16,1 / 2$

278 colour judgement environments. Imagemaster is often used as a Colour Library and historical colour database (with no limit on the number or type of objects). Another example is the use of Imagemaster for colour CAD discussions. It can then support the designer with a database of historical colour-popularity data, and future popularity predictions such as those available from the International Colour Council.

For all active objects in a screen simulation, a pop-up window providing feedback on the colour data is available, giving both CIE co-ordinate and spectral definitions. The graphical data thus provided make it easy to study the difference between selected objects and colours. If more than one object in the system is selected, the colour difference between each is displayed (using SPD data to define the current illuminant simulation, and a range of colour-difference equations).

\section{The advantages of using calibrated colour images}

There are a number of important consequences following the adoption of CIE specified colour, for image definition and processing.

(1) It becomes possible (and often necessary) to define simulated colour with reference to input measured colour specifications, and also to compare the on-screen simulation with the physical samples originally measured.

(2) It is possible to visualise remotely generated colour specifications and texture simulations.

(3) Intrinsic colour differences and internal colour relationships within images can be captured, analysed and used to simulate and define known textures.

(4) When image colour-sets are moved as a body within CIE $L * C * H *$ or CIE $L * a * b *$ colour space, internal colour relationships are preserved to a great extent. It is thus possible to move an entire image colour-set quite a long way inside $3 \mathrm{D}$ colour-space, thus re-colouring the object without losing correct texture and reproducible appearance.

(5) CIE-based colorimetric analysis is demonstrated as a very powerful method of abstracting logical object hierarchies from images, and also for advanced colour reduction strategies in print design.

\section{Characterizing and modelling texture}

A definition of texture can be established based on the detailed (sub-millimetre level) spatial distribution of coloured areas within an image. The phenomenon of "colour appearance" is then modelled as having two independent variables. They are the "texture" and its intrinsic base-colour (i.e. single colour) specification. The base colour of a texture then becomes an independent variable which can be changed at will, and is a direct analogue of applying a dye to a fabric with the given constant texture. 
The independent variable called "texture" has two components. Each colour definition has a frequency of occurrence (by total pixel count), and a set of locations at which this colour is present, distributed spatially across the texture.

The first component is a mapping from the base-colour specification into a cluster of closely related colour specifications. The second component establishes the spatial-position of each pixel-member of the cluster, to represent the characteristic visual appearance of the texture.

In practice, this two-part definition leads to a simulation of texture as a set of possibly many thousand colour definitions, called a "colour-set". Colour sets within excess of 100,000 colour definitions, and perhaps millions of spatially related pixel positions, occur frequently in high resolution image analysis.

Modern PCs with a Pentium 3 processor or higher and sufficient memory are powerful enough to handle the necessary calculations for creating, storing and processing the required data structures.

\subsection{MDD and MVD value quantification of "texture"}

A mathematical and statistical analysis has been made, of object and texture colour-sets abstracted from images, and the resulting colour-sets have been characterised by a new scalar measure of colour distribution within each colour-set. This has been given the name "mean directional deviation" (MDD), and it relates colour-set members to the base-colour by means of a three-dimensional mathematical function. The MDD in practice is related to the common experience that an identical dye recipe may produce a shade with perhaps lighter or more vibrant colour in certain textures.

As the members of a colour-set are distributed in a colour-space to which vector properties can be ascribed, a second powerful measure of the properties of a texture colour-set becomes available. It has been given the name "mean vector displacement" (MVD) value. The adopted "independent texture variable" is thus defined as a function which operates on an base-colour definition to produce a modified colour appearance. The MVD value specifies the result of applying the function, and thus has both diagnostic and predictive power for changes in visual appearance due to changes in texture.

\subsection{The derivation of MDD and MVD values}

The MDD of a colour-set in each of the three dimensions is defined as the mean sum of all the (many thousand) individual point-colour deviations:

for example, in the lightness dimension

$$
\mathrm{MDD}_{\mathrm{L}}=\left(\sum \Delta L\right) / n
$$

where $n$ is the number of set members, and $\Delta L$ is an individual point-colour lightness difference from the intrinsic colour $L^{*}$ value indicated by the dye recipe prediction. 
IJCST

$16,1 / 2$

\section{0}

$\mathrm{MDD}_{\mathrm{L}}$ defines the MDD of all the members of a colour-set from the intrinsic colour in the lightness dimension of difference. It is measured in colour-difference units i.e. CIE $L * a * b *$ units.

Identical measures, $\mathrm{MDD}_{\mathrm{C}}$, and $\mathrm{MDD}_{\mathrm{H}}$ are also defined for the hue and chroma dimensions of difference.

$\mathrm{DD}_{\mathrm{L}}$, the direction of deviation in lightness, is a basis-vector of three-dimensional colour-difference space. The other two basis-vectors are $\mathrm{DD}_{\mathrm{C}}$ and $\mathrm{DD}_{\mathrm{H}}$, respectively, in the dimensions of chroma and hue. Individual set-member deviations, and mean colour-set deviations MDD, are scalar measures in this colour space, which are regarded as having both direction and magnitude.

Directional hue difference $\Delta H_{\mathrm{D}}$ is a signed scalar variable in this space, defined as

$$
\Delta H_{\mathrm{D}}=\Delta H^{*}\left(\Delta h^{\circ} /\left|\Delta h^{\circ}\right|\right)
$$

where $\Delta H$ is the metric hue difference (which does not have a defined direction of change), $\Delta h^{\circ}$ is the signed hue angle change, and $\left|\Delta h^{\circ}\right|$ is its un-signed absolute value.

The effect of the function $\left(\Delta h^{\circ} /\left|\Delta h^{\circ}\right|\right)$ is to assign a direction of change \pm to $\Delta H$ without altering its numeric value.

\subsection{MVD}

The three mean-value scalars are combined to produce a colour appearance-change vector in the three dimensions of colour: lightness chroma and hue ( $L C$ and $H$ ). It is denoted by the symbol MVD Texture.

$$
\mathrm{MVD}_{\text {Texture }}=\left(\mathrm{MDD}_{\mathrm{L}}, \mathrm{MDD}_{\mathrm{C}}, \mathrm{MDD}_{\mathrm{H}}\right) \text {. }
$$

Because the MVD is regarded as a vector in colour-difference space, it is appropriate to quote a colour difference between two MVDs in this space. This difference is denoted as delta $E_{\mathrm{MVD}}$. It is calculated in the same way as conventional colour difference delta $E$ (i.e. as an RMS value) as follows.

$$
\text { delta } E_{\mathrm{MVD}}=\left(\mathrm{MDD}_{\mathrm{L}}^{2}+\mathrm{MDD}_{\mathrm{C}}^{2}+\mathrm{MDD}_{\mathrm{H}}^{2}\right)^{1 / 2}
$$

The difference expressed in delta $E_{\mathrm{MVD}}$ (and also in a single MVD value), is defined in terms of CIE $L * a * b *$ colour difference units. In effect, delta $E_{\mathrm{MVD}}$ establishes the overall size of the effect produced by the texture relative to the base colour, and the MVD establishes the direction of difference.

The MVD is in principle constant over a large range of intrinsic colours for a given texture. The colour-set, from whose colour definitions MVD values are calculated, is an analogue of a textured substrate, to which any intrinsic base-colour can be applied. The base colour is then an analogue of a colourant formulation that might be applied to the substrate. 
The MVD for a given texture, plus a base-colour defined by a reflectance curve, combine to give a direct simulation and quantification of any colour appearance difference attributable to texture.

The validity and independence of MVD values have been demonstrated (Oulton et al., 1998) in a series of multi-observer trials where it successfully predicted the appearance of a dyed textile yarn, when viewed sequentially as a yarn winding, a knitted fabric, and a cut tuft, in 20 different intrinsic colours.

MDD and MVD values were used in a different context to test their validity as a constant characteristic of 63 similar instances of a constant texture. The trials established (Oulton and Porat, 1997) that calculated MDD and MVD values are consistent across the 63 instances, to the limits of experimental error (Figure 1).

MDD and MVD values encapsulate the vector principle of mapping multi-dimensional variables (in the above case colour-set membership) onto a meaningful three-dimensional definition of their effect (displacement of colour identity in colour-space). The process is analogous to the common experience of colour matches that only work under certain lighting conditions. Only textures with the same MVD will dye to the same shade using the same dye recipe.

In the current context, the prediction is for textures that will have the same visual colour appearance when they are dyed with the identical dye recipe.

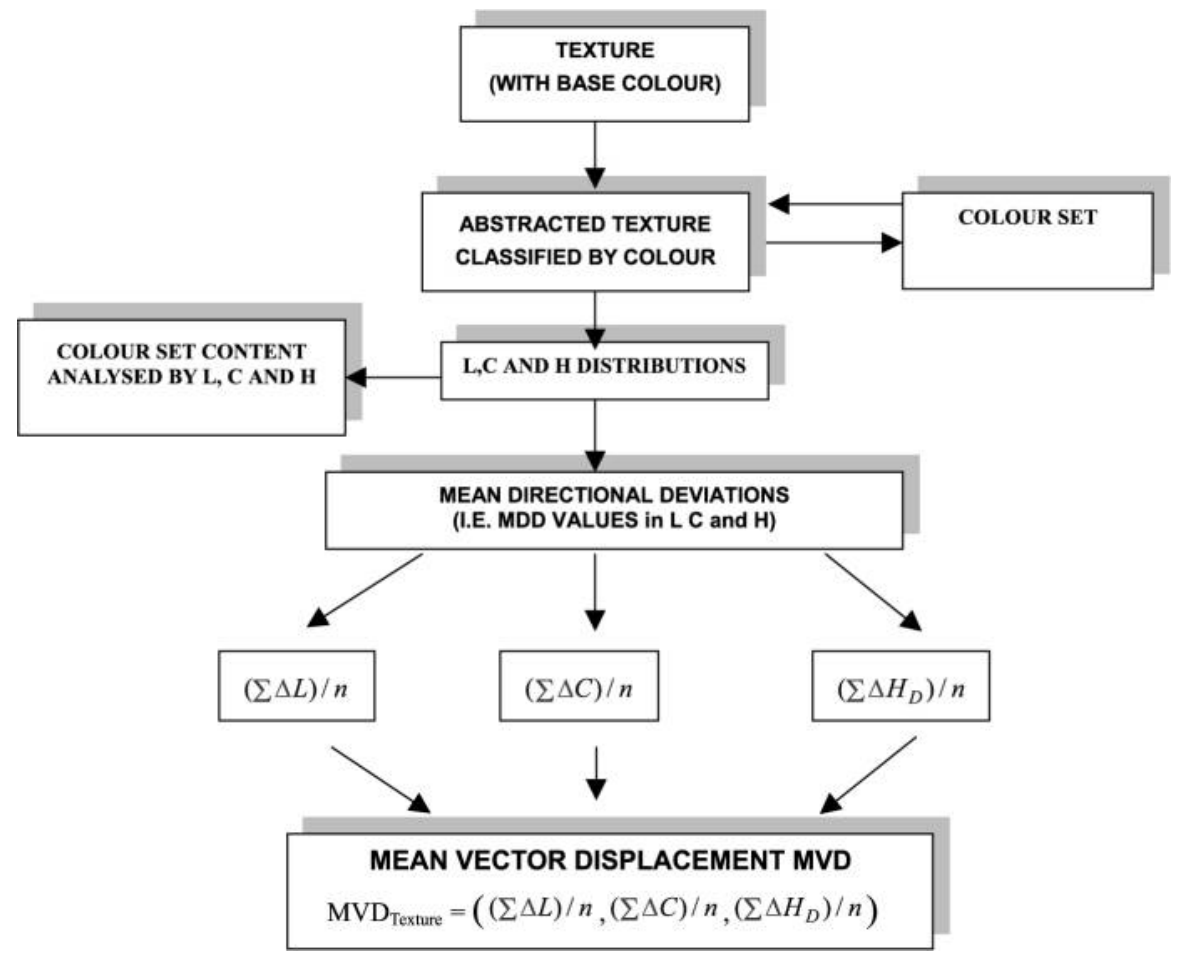

Figure 1. Derivation of MDD and MVD values 


\section{IJCST}

$16,1 / 2$

\section{2}

\section{Figure 2.}

Pixel population

distributions. A

yellowish green medium depth, medium chroma image of a textile yarn winding is analysed that is judged by observers to have a single intrinsic colour

\section{Figure 3.}

Pixel population distributions. A

yellowish green medium depth, medium chroma image of a textile yarn winding is analysed that is judged by observers to have a single intrinsic colour

\subsection{Colour-set populations}

An example of a colour-set is illustrated later. It has been isolated from a high resolution photograph using a three-dimensional lightness-, chroma- and hue- $(L, C, H)$ based segmentation algorithm. The extracted colour-set, representing a green textile yarn winding, is analysed in Figures 2-4. RGB integer-value quantization in the screen-drive values is evident in the graphs.
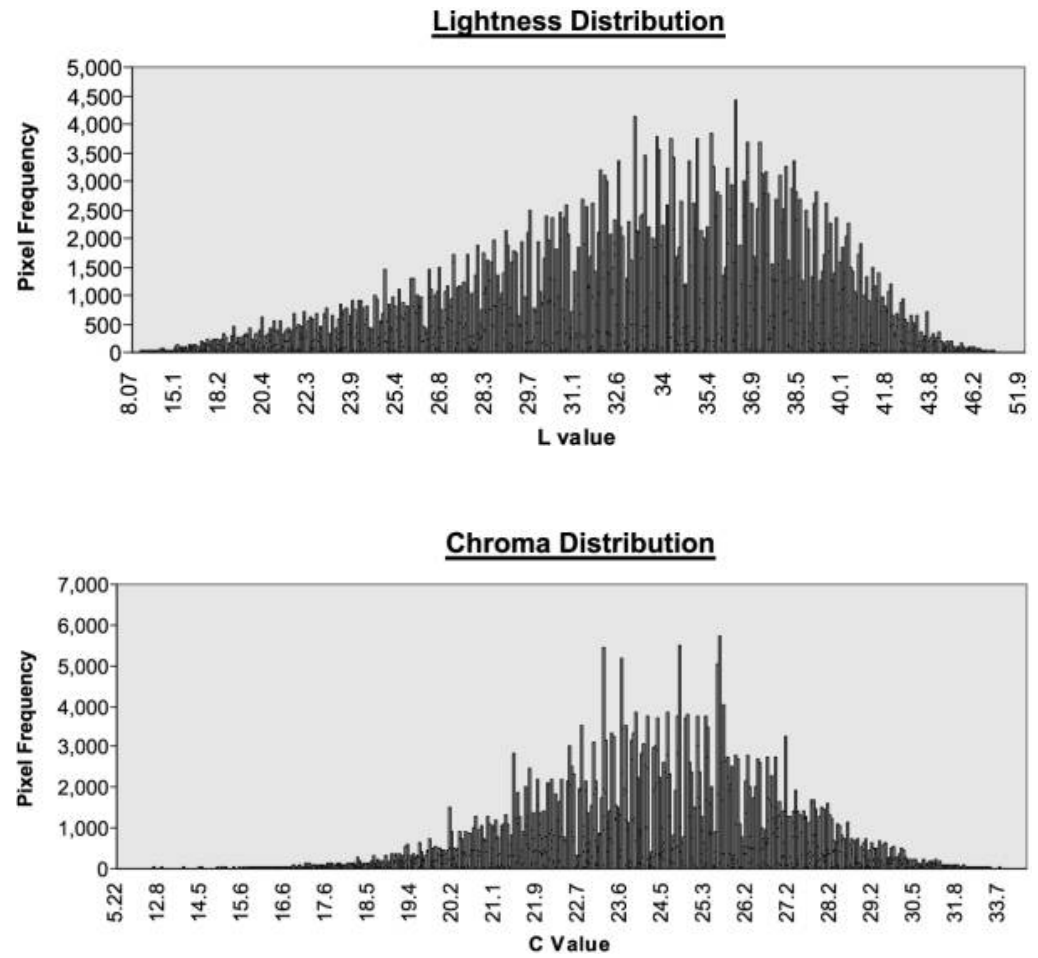

Figure 4.

Pixel population distributions. A yellowish green medium depth, medium chroma image of a textile yarn winding is analysed that is judged by observers to have a single intrinsic colour

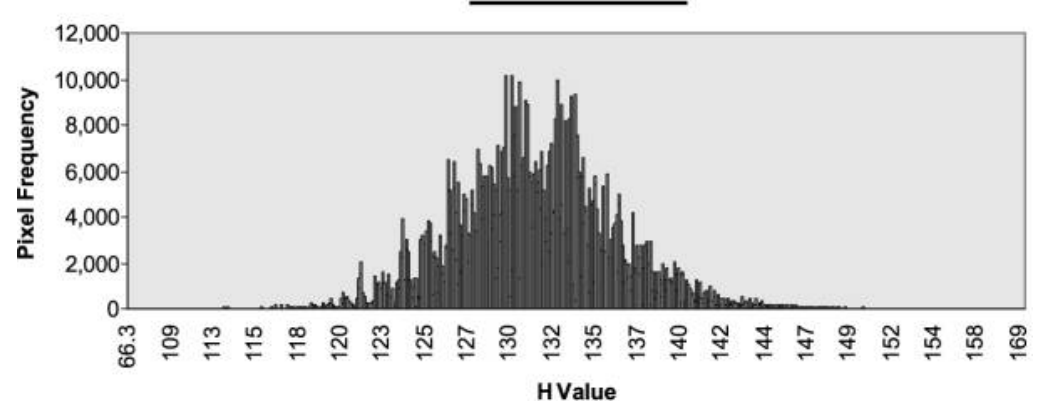


The quantization effect is responsible for the evident "high frequency noise" in the otherwise smooth frequency distributions in $L, C$ and $H$ of the individual pixels.

The observers rated the image to be a visual match to the physical sample, and also rated its appearance as a texture with a single intrinsic colour. The relevant pixel populations in the colour-set are graphed by pixel count on the $Y$ axis in Figures 2-4 as separate $L, C$ and $H$ distributions.

Interestingly, both the hue and chroma distributions are significantly bi-modal. This in fact represents a detailed difference between highlight and shadow colour. It is also the fundamental reason why injecting colour into "black and white" (i.e. grey-scale) images often produces an artificial or flat appearance in the resulting coloured image.

The effect is usually identifiable in textures built up from essentially transparent or translucent coloured materials, particularly if they are also glossy. Textile fibrous assemblies are a good example.

\section{Imagemaster in an industrial context}

For the past 2 years, a "Teaching Company" partnership between UMIST and Worthington Manufacturing Ltd has been used, to enable and establish Imagemaster links between the company and its suppliers and customers in the Lingerie Industry (Oulton and Young, 2003). The key tasks included developing an understanding of the opportunities and limitations of the concepts of calibrated colour visualization. They also include introducing both company and customer personnel to the new technology of digital colour communication. Both practical and conceptual familiarization was found to be necessary.

An important outcome is a marked reduction of delays and cost associated with the production of physical lab-dye samples and their communication by postal methods. An equally important outcome is the formation of much better supplier/buyer collaborative teams for product development. In the longer term this produces both continuity of orders and a clear competitive edge when the communication link is with design-lead buying teams who specify the lingerie sets and garments containing components supplied by Worthington Manufacturing Ltd.

The Colorite Division of Datacolor Inc. (Datacolor, 2003) supply Imagemaster as a commercial system, and they have sold it to about 200 companies in over 40 countries across five continents, working in the cosmetic, automotive, paint, plastic, and retail industries as well as in textiles.

It is clear that the problem of "Colour Specification at the Design to Production Interface" is widely encountered, and a solution based on calibrated digital colour imaging has a wide general appeal. 
IJCST

$16,1 / 2$

\section{4}

\section{References}

Berns, R.S. (2000), Billmeyer and Saltzman's Principles of Color Technology, 3rd ed., Wiley-Interscience, New York, NY.

Datacolor (2003), Datacolor Headquarters: 5 Princess Road Lawrenceville, NJ 08648 USA.

Hawkyard, C.J. (1993), "Synthetic reflectance curves by additive mixing”, $\underline{S D C}$, Vol. 109, p. 323.

Oulton, D.P. and Porat, I. (1991), “Control of colour using measurement and feedback”, J.Text. Inst., Vol. 83 No. 3, p. 453.

Oulton, D.P. and Porat, I. (1997), Final Report EPSRC Project "Colour Systems for Texture in Textile CAD”, No. GR/J91852 1994-96 (The Imagemaster Project), UMIST, Manchester.

Oulton, D.P. and Young, T. (2003), "Accurate colour co-ordination for the textile components of lingerie", Proc. Int. Conf. CESA 2003 "Computational Engineering in Systems Applications", University of Beaulieu, France.

Oulton, P., Boston and Walsby (1996), "Imagemaster: precision colour communication based on CIE calibrated monitor screens", Proc. 5th Int. Conf. High Technology, Chiba, Japan, p. 290.

Oulton, D.P., Peterman, E. and Bowen, A.W. (1998), "Measuring the contribution of texture to colour appearance", in Nobbs, J. (Ed.), Proc. Int. Conf. Colour 98, April 1998, Harrogate, Department of Colour Chemistry, Leeds Univ., ISBN 085316218 2, pp. 12-21.

Smith, K.J. (1999), “A revolution in colour communication”, January International Dyer, p. 12.

\section{Further reading}

Sargeant, C. (1999), "Colour visualization and communication - a personal view”, in Dinsdale, P. (Ed.), Rev. Prog. Coloration, Soc Dyers and Colourists, Vol. 29, pp. 65-70. 
This article has been cited by:

1. D.J. TylerDigital printing technology for textiles and apparel 259-282. [CrossRef]

2. Samo Kreft, Marko Kreft. 2009. Quantification of dichromatism: a characteristic of color in transparent materials. Journal of the Optical Society of America A 26:7, 1576. [CrossRef]

3. David J. Tyler. 2005. Textile Digital Printing Technologies. Textile Progress 37:4, 1-65. [CrossRef] 